\title{
Targeted Accrual at Clinical Study Site
}

National Cancer Institute

\section{Source}

National Cancer Institute. Targeted Accrual at Clinical Study Site. NCI Thesaurus. Code C70789.

A total number of subjects needed for the entire clinical study at a particular clinical study site. 